\title{
Experimental Study on Gas Explosive Limits Regularities under Coupling Effects of Temperature and Pressure
}

\author{
ZHU Pi-kai ${ }^{1,2}$ \\ (1.Shandong University of Science and Technology, Qingdao, Shandong,266510; 2 China Coal \\ Technology Engineering Group Chongqing Research Institute Co., Ltd., Chongqing 400037, \\ China)
}

Keywords: coupling effects of temperature and pressure, gas explosive limit, variation law, experimental study

\begin{abstract}
Gas explosion is a kind of serious natural disaster in coal mines. In order to prevent gas explosion accidents, it is necessary to study the characteristics of gas explosion under different temperature and different pressure conditions. In this paper the influence of gas explosive limits under coupling effects of temperature and pressure was studied. The variation trend of gas explosive limits along with temperature and pressure was obtained. By comparing the theoretical and experimental results, it shows that the gas explosive limit value obtained from the experiment is close to the theoretical value, and with the change of environment temperature and pressure, they have the similar variation laws. So the experimental results of gas explosive limits obtained are reasonable and accurate.
\end{abstract}

\section{Introduction}

In recent years, gas explosion accidents in China's coal mine occur frequently, causing huge losses to state property and people's life. Therefore, there is important significance to study the gas explosive limit on the prevention of coal mine gas accident.

Many factors that impact on gas explosive limit were studied in the experimental studies,such as: mixed gases, initial temperature, initial pressure, coal dust and state of gas, etc. but study on the coupling effect of temperature and pressure on gas explosive limit is seldom reported. In this paper, variation laws of gas explosive limit under different environment conditions were studied by experiment.

\section{Experimental situation}

To study the gas explosive limit under special environment, the same equipment, the same test procedure, and the same parameters were used, meanwhile, the environment temperature and environment pressure were changed.

The experiments were conducted under the environment conditions of $50 \sim 200^{\circ} \mathrm{C}$ and $0.2 \sim 1.0 \mathrm{MPa}$, and using the $10 \mathrm{~J}$ electric spark as the test ignition source. The specific test conditions as shown in table 1 . 
Table 1 Gas explosion experimental conditions

\begin{tabular}{ccccc}
\hline $\begin{array}{c}\text { Initial } \\
\text { pressure } \\
/ \mathrm{MPa}\end{array}$ & $\begin{array}{c}\text { Initial } \\
\text { temperature } \\
/{ }^{\circ} \mathrm{C}\end{array}$ & $\begin{array}{c}\text { Environmnetal } \\
\text { relative humidity } \\
/ \% \mathrm{rh}\end{array}$ & $\begin{array}{c}\text { Ignition } \\
\text { energy } \\
/ \mathrm{J}\end{array}$ & $\begin{array}{c}\text { Gas state before } \\
\text { explosion }\end{array}$ \\
\cline { 1 - 4 } $0.2 \sim 1$ & $\begin{array}{c}\text { Room } \\
\text { teperture } \sim 200^{\circ} \mathrm{C}\end{array}$ & $60 \sim 90$ & 10 & Static state \\
\hline
\end{tabular}

\section{Experimental results and analysis}

Under the experimental conditions the upper explosive limit and the lower explosive limit of gas explosion were obtained by using the same separately testing method.

\section{The coupling effect of temperature and pressure on the upper limit of gas explosion}

According to the experimental data, the variation law of the gas explosive upper limit with the environment temperature and pressure were respectively fitted to histograms, as shown in Fig. 1 and Fig.2.
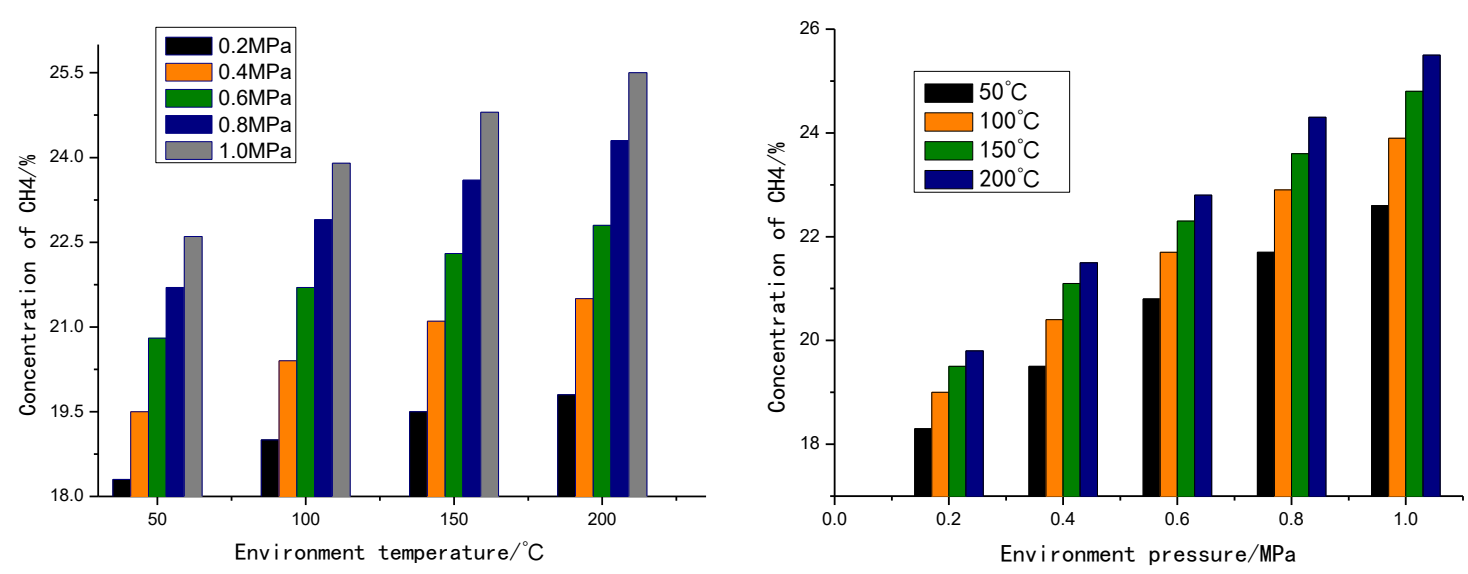

Fig.1 Gas upper explosive limit curves with temperature Fig.2 Gas upper explosive limit curves with pressure

It can be seen from the graphs that the gas upper explosive limit is index trend under the coupling effect of environment temperature and pressure. Seen from Fig.1, under the same environment pressure conditions, the gas upper explosive limit increases with the rising of temperature, and under the same temperature, the gas upper explosive limit becomes higher when the pressure is greater. As can be seen from Fig.2, in the condition of the same environment temperature, the gas upper explosive limit increases along with the pressure increases, and under the same pressure, the gas upper explosive limit becomes higher when the temperature is greater.

Set the environment temperature and pressure as the $\mathrm{X}$ axis and $\mathrm{Y}$ axis, and the gas upper explosive limit as $\mathrm{Z}$ axis, the gas upper explosive limit surface was obtained as shown in Fig.3. 


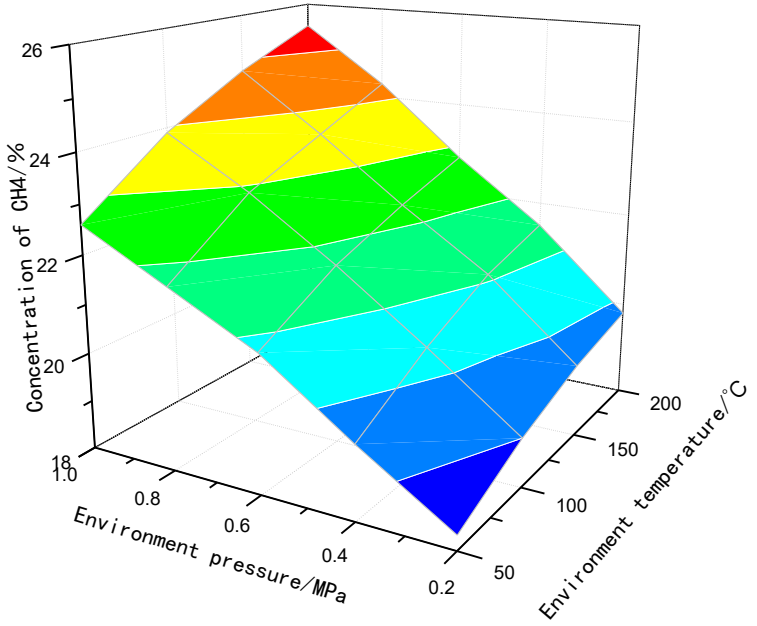

Fig.3 Gas upper explosive limit surface under coupling conditions

From Fig. 3 the variation trend of gas upper explosive limit under the coupling conditions can be seen directly. And according to Fig.3, a Gauss equation of gas upper explosive limit changed with the environment temperature and pressure was obtained as follows.

$$
Z=Z_{0}+A * \exp \left[-\frac{1}{2}\left(\frac{T-T_{0}}{w_{1}}\right)^{2}-\frac{1}{2}\left(\frac{P-P_{0}}{w_{2}}\right)^{2}\right], \quad\left(50 \leq T \leq 200^{\circ} \mathrm{C} \quad, \quad 0.2 \leq P \leq 1.0 \mathrm{MPa}\right)
$$

Comparisons of fitting function under coupling conditions are shown in table 2.

Tab. 2 Comparison table of fitting function under coupling conditions

\begin{tabular}{cccccccc}
\hline parameter & $Z_{0}$ & $A$ & $T_{0}$ & $w_{1}$ & $P_{0}$ & $w_{2}$ & $R^{2}$ \\
\hline value & 12.693 & 14.20653 & 1.47912 & 1.1098 & 251.27623 & 278.59274 & 0.99812
\end{tabular}

Experimental research under the certain temperature $\left(50 \sim 200^{\circ} \mathrm{C}\right)$ and pressure $(0.2 \sim 1.0 \mathrm{MPa})$ shows that with the rising of the environment temperature and pressure, the gas upper explosive limit increases. But the upper explosive limit values will not increase infinitely, when the gas density increases to a certain value, there will be not enough oxygen to sustain the reaction, the reaction environment will be in the negative oxygen state, no matter how the environment conditions change to enhance the strength of gas explosion, no explosion will happen.

\section{The coupling effect of temperature and pressure on the lower limit of gas explosion}

According to the experimental data in Tab.2, the variation law of the gas explosive lower limit with the environment temperature and pressure were respectively fitted to histograms, as shown in Fig.4 and fig.5.
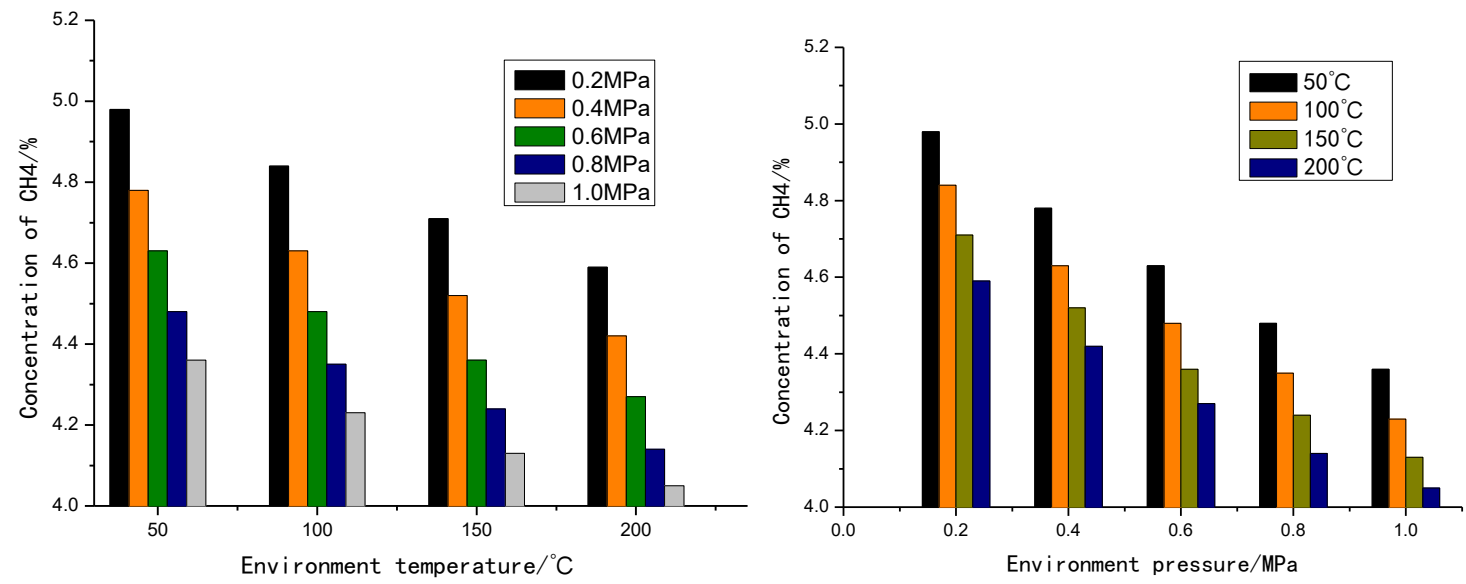

Fig.4 Gas lower explosive limit curve with temperature Fig.5 Gas lower explosive limit 
curve with pressure

It can be seen from the graphs that the gas lower explosive limit is index trend under the coupling effect of environment temperature and pressure. Seen from Fig.4, under the same environment pressure conditions, the gas lower explosive limit declines along with the temperature increases, and under the same temperature, the gas lower explosive limit becomes lower when the pressure is greater. As can be seen from Fig.5, in the condition of the same environment temperature, the gas lower explosive limit declines along with the pressure increases, and under the same pressure, the gas lower explosive limit becomes lower when the temperature is greater.

Set the environment temperature and pressure as the $\mathrm{X}$ axis and $\mathrm{Y}$ axis, and the gas lower explosive limit as $\mathrm{Z}$ axis, the gas lower explosive limit surface was obtained as shown in Fig.6.

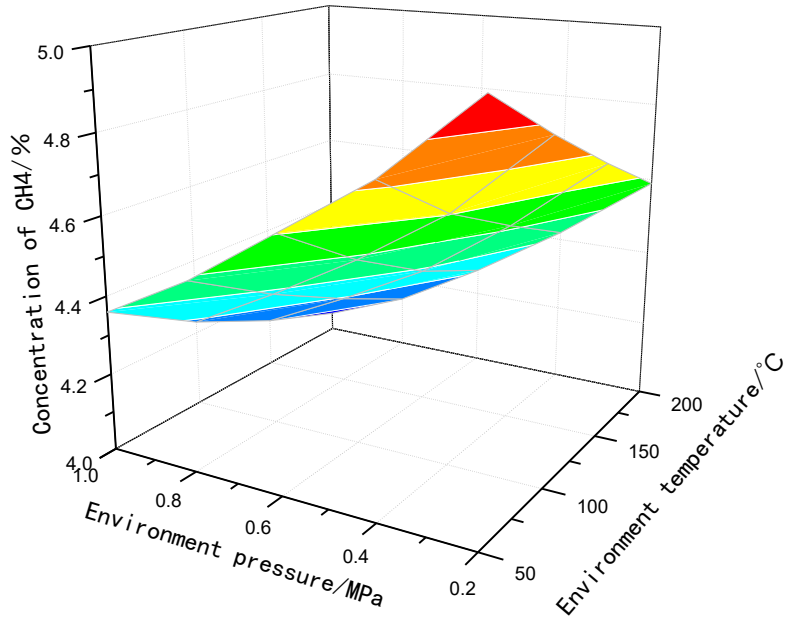

Fig.6 Gas lower explosive limit surface under coupling conditions

From Fig.6 the variation trend of gas lower explosive limit under the coupling conditions can be seen more directly. And according to Fig.6, a Gauss equation of gas lower explosive limit changed with the environment temperature and pressure was obtained as follows.

$$
Z=Z_{0}+A * \exp \left[-\frac{1}{2}\left(\frac{T-T_{0}}{w_{1}}\right)^{2}-\frac{1}{2}\left(\frac{P-P_{0}}{w_{2}}\right)^{2}\right], \quad\left(50 \leq T \leq 200^{\circ} \mathrm{C} \quad, \quad 0.2 \leq P \leq 1.0 \mathrm{MPa}\right)
$$

Comparisons of fitting function under coupling conditions are shown in tab.3.

Tab.3 Comparison table of fitting function under coupling conditions

\begin{tabular}{cccccccc}
\hline parater & $Z_{0}$ & $A$ & $T_{0}$ & $w_{1}$ & $P_{0}$ & $w_{2}$ & $R^{2}$ \\
\hline value & 1063.4 & -1059.7 & 1.70405 & 40.019 & 403.39206 & 11224.4 & 0.99724 \\
\hline
\end{tabular}

Experimental research under the certain temperature $\left(50 \sim 200^{\circ} \mathrm{C}\right)$ and pressure $(0.2 \sim 1.0 \mathrm{MPa})$ shows that with the rising of the environment temperature and pressure, the gas lower explosive limit declines. But the lower explosive limit values will not decline infinitely. When the gas density is less than a certain value, there will be not enough gas to sustain the reaction, the reaction environment will be in the rich oxygen state, no matter how to encourage the activity of gas molecules, no explosion will happen.

\section{Comparison between theoretical results and experimental results of gas explosive limits}

According to the previous theoretical analysis, assuming that under the coupling condition of environment temperature and pressure, the gas upper and lower explosive limits are shown as the following formulas:

$$
U=\sqrt{\left[U_{25}+8 \times 10^{-4} U_{25}(t-25)\right]\left[U_{25}+20.6(\log p+1)\right]}
$$




$$
L=\sqrt{\left[L_{25}-8 \times 10^{-4} L_{25}(t-25)\right]\left[L_{25}-0.71 \ln (10 p)\right]}
$$

Under standard atmospheric pressure at $25{ }^{\circ} \mathrm{C}$ the gas upper and lower explosive limits are calculated respectively according to the following data: $U_{25}=16.0 \%, L_{25}=5.0 \%$, and according to the formula (3) and (4), the theoretic values of gas upper and lower explosive limits under coupling conditions can be obtained respectively.

Comparison between theoretical values and test values of gas upper and lower explosive limit was shown in Fig.7and Fig.8, along the $\mathrm{X}$ axis forward direction, the increasing environment pressure of 5 data in each curve are respectively: $0.2 \mathrm{MPa}, 0.4 \mathrm{MPa}, 0.6 \mathrm{MPa}, 0.8 \mathrm{MPa}$ and $1.0 \mathrm{MPa}$.

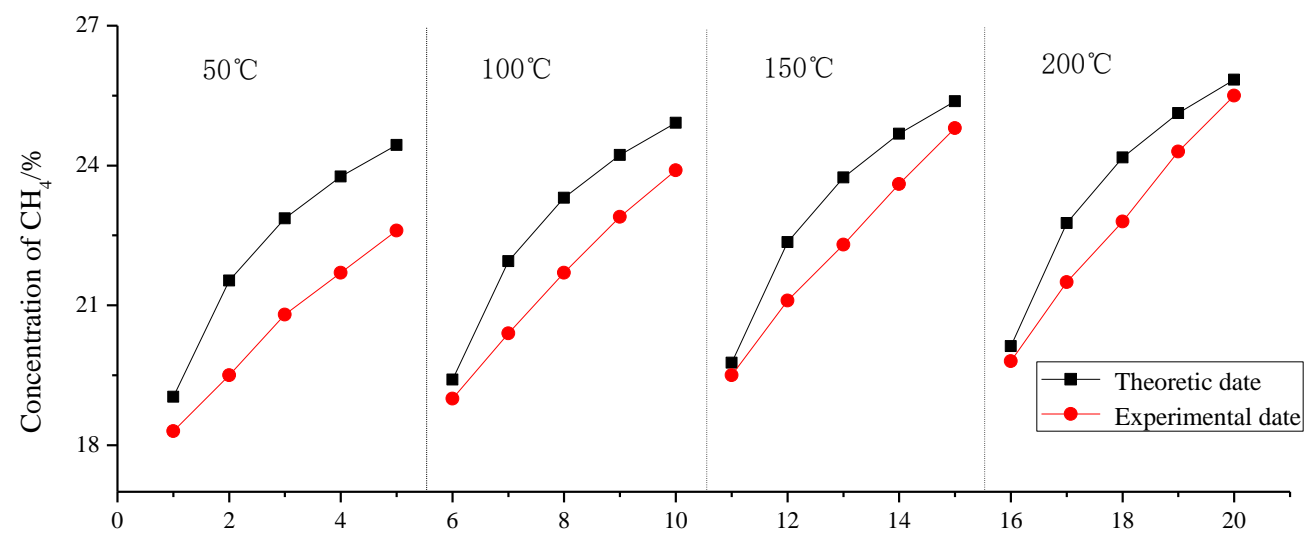

Fig.7 Theoretical values and test values of gas upper explosive limit

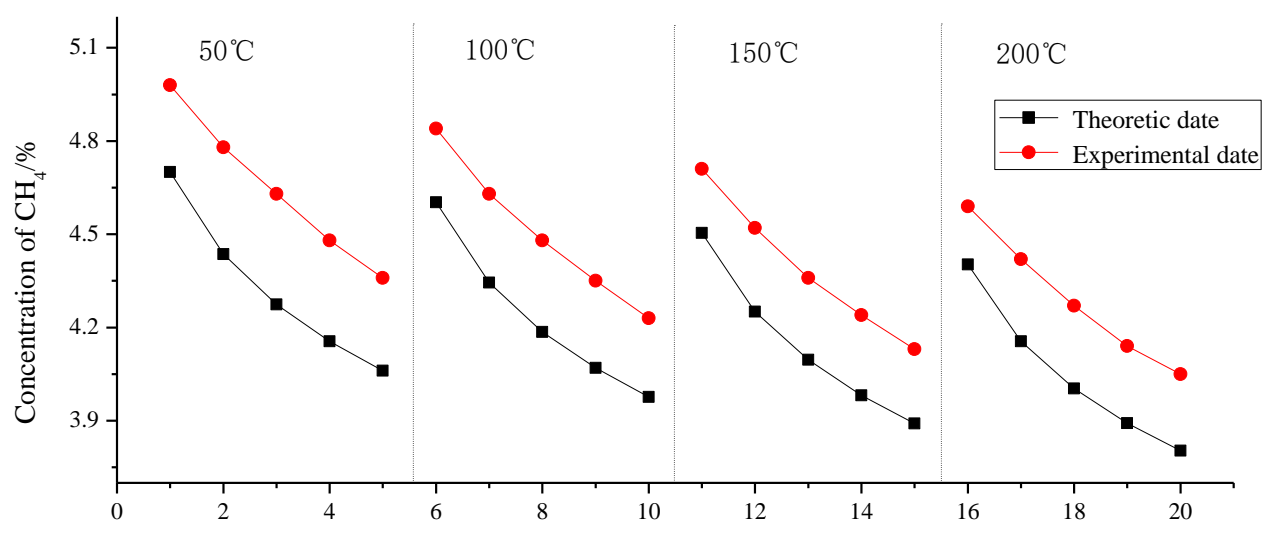

Fig. 8 Theoretical values and test values of gas lower explosive limit

It can be seen that with the rising of the environment temperature and pressure, the gas upper explosive limit increases and the lower explosive limit declines, and the explosive range widens. Compared the theoretical results and the experimental results of gas explosive limits, we found that they are in good agreement with each other. It is to say that our experiment study results are reasonable and accurate.

\section{Conclusions}

In this paper the influence of gas explosive limits under coupling effects of temperature and pressure was studied by experiments. The variation trend of gas explosive limits along with temperature and pressure was obtained. The main conclusions are as follows:

(1) With the rising of the environment temperature and pressure, the gas upper explosive limit increases and the lower explosive limit declines, and the explosive range widens, which means the 
risk of gas explosion increases.

(2)By comparing the theoretical results and the experimental results of gas explosive limits, we found that they are in good agreement with each other. It is to say that our experiment study results are reasonable and accurate.

\section{References}

[1]Li Runzhi, Si Rongjun, Zhang Yansong, Wang Lei. Research Status and Development Direction of Characteristics of Gas Explosion[J]. Coal Technology, 2010, 29(4):4-6.

[2] Li Gang, Li Yufeng,Yuan Chunmiao. Explosion Limits of CBM at Elevated Pressure and Temperature [J]. Journal of Northeastern University: Natural Science,2012,33(4):580-583.

[3]Yao Jie,Jiang Juncheng, Pan Yong. The Effect of Initial Temperature on Lower Explosion Limit of Flammable Gas [J]. Industrial Safety and Environmental Protection,2012,38(2): 48-50.

[4] Li Runzhi, Si Rongjun. Experimental Study on Impact of Ignition Energy on Gas Explosion Pressure[J].Mining Safety \& Environmental Protection, 2010,37(2): 14-20.

[5]Yang Zhao,Wang Xiaoming, WU Xi,et al. Explosion Limits of Flammable Refrigerants Under Condition of Variable Temperature [J]. Journal of Tianjin University,2012,45(10):851-854

[6] Hou Wanbing, Tan Yingxin, Zhang Yinghao. Experiment and Study on Gas Explosion Features in Simulated Mine Roadway[J]. Coal Science and Technology,2009,37(5):58-61. 